\title{
THE MAGNETIC DESIGN AND PERFORMANCE OF THE SRRC-U9 UNDULATOR
}

\author{
S.C. Gottschalk, D.C. Quimby, K.E Robinson* \\ STI Optronics, Inc., Bellevue, WA 98004 USA \\ *Presently with Lawrence Berkeley National Laboratory.
}

\begin{abstract}
The magnetic design of the recently completed SRRC-U9 undulator system employs several enhancements to improve its performance. Side magnets to increase onaxis field augment the wedged-pole ${ }^{1}$ magnet geometry. Partial volume and partial strength end magnets and tuning bars achieve a zero-displacement, rapid turn-on, end-field configuration. ${ }^{2}$ Gap dependent normal and skew component ambient (earth) field compensation has been included in the design. The design includes bot active and passive end correction. Optical phase and trajectory shimming techniques have been employed to optimize performance. For $\mathrm{k}=10$ the performance is greater than $80 \%$ of ideal through the seventh harmonic. At a $\mathrm{K}=6.4$ the calculated spectral performance is greater than $80 \%$ through the thirteenth harmonic. A complete battery of magnetic measurements has fully established the field, spectral and beam dynamic characteristics of the undulator.
\end{abstract}

\section{INTRODUCTION}

This paper describes the magnetic design of the U9 undulator. ${ }^{3}$ It is a wedged pole hybrid planar insertion device built for the Synchrotron Radiation Research Company located in Hsinchu, Taiwan, R.O.C. A detailed discussion of the magnetic design method that we use has been described elsewhere. ${ }^{4}$ This device had three new features: 1) side magnets which were added to increase the peak field, 2) non-displacing entrance and exit built by using partial strength and reduced volume end magnets and 3) pre-tuning to remove ambient field differences between the STI Optronics site in Seattle, Washington, and SRRC.

\section{CENTRAL FIELD}

Wedged poles hybrids were used to generate the high fields required by SRRC with $20 \%$ less magnet volume than a straight pole. In addition, wedged magnets were small enough to permit single piece fabrication, unlike straight sided magnets. An isometric view of a quarter period cell is shown in Figure 1. The wedge angle, chamfers and magnet recess were chosen to remove saturated parts of the pole, reduce demagnetizing fields, allow sufficient space for tuning shims and control higher field harmonics. ${ }^{5}$ The design approach was conservative: low remanence magnets were used for peak field estimates but high remanence was used for pole saturation, transverse rolloff and parts tolerancing. This had two ramifications: 1) side magnets are needed for field strength margin, and 2) pole transverse width was increased to $8.0 \mathrm{~cm}$ to keep the transverse rolloff small $(0.035 \%$ at $\pm 10 \mathrm{~mm}$ was calculated), in the event that stronger magnets were delivered by the vendor.

Adding side magnets reduced transverse flux leakage to $0.5 \%$ without degrading the transverse rolloff, changing field harmonics or reducing the pole permeability. For ease of fabrication we selected a side magnet that increased the field strength $5.2 \%$. Larger side magnets could have increased the field by $8 \%$. While the side magnets did have clamps, the lack of a transverse overhang caused them to be so strongly attracted to the poles that special tooling was needed to remove them during end field adjustment. Our simulated annealing magnet sorting algorithm was modified to include steering and multipole moments that can be created by side magnets. Only a small, easily removed quadrupole was observed on the untuned device. When side magnets are properly sorted, they do not appear to degrade field errors.

Table 1: Comparison of magnet predictions with measured performance.

\begin{tabular}{|l|c|c|c|}
\hline Quantity & Specification & MAGNET FEA & Experiment \\
\hline Peak field, g=18mm & $1.245 \mathrm{~T}$ & $1.365 \mathrm{~T}$ & $1.365 \mathrm{~T}$ \\
\hline Transverse rolloff at $10 \mathrm{~mm}$ & $<0.1 \%$ & $0.035 \%$ & $0.037 \%$ \\
\hline $3^{\text {rd }}$ Harmonic content & $<10 \%$ & $5.8 \%$ & $5.8 \%$ \\
\hline
\end{tabular}

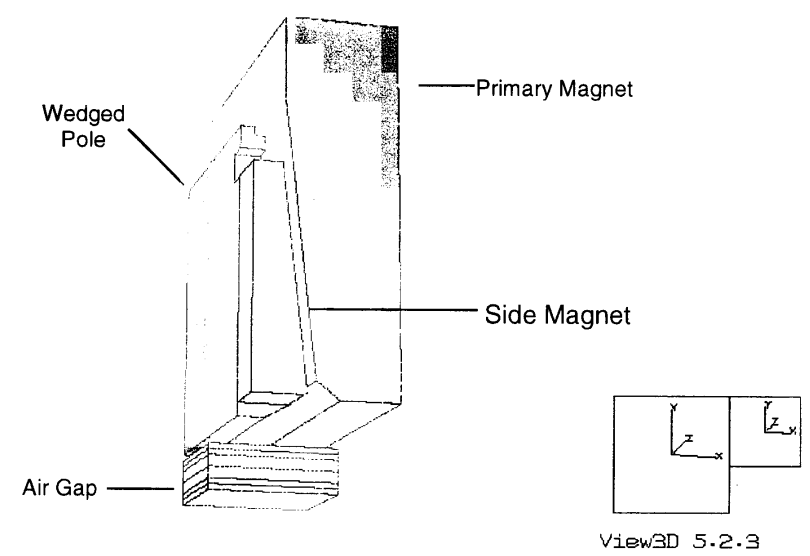

Figure 1: Isometric view of quarter period cell. 


\section{AMBIENT FIELD}

Hybrid ID's can modify any ambient fields that are present causing gap dependent changes in beam dynamics which could require retuning the device at the new location. The following hypothetical example illustrates the point. Site A has a 0.2 Gauss ambient while site B has a 0.6 Gauss ambient. The ID $450 \mathrm{~cm}$ long and has no $1^{\text {st }}$ or $2^{\text {nd }}$ integral. Normal field enhancement is about 1.8 at minimum gap. For site A the ambient field inside the ID is increased to $1.8 * 0.2=0.36$ Gauss and the $1^{\text {st }}$ integral changes by $(0.36-0.2) * 450=72 \mathrm{G}-\mathrm{cm}$ while the $2^{\text {nd }}$ has changed by $16,200 \mathrm{G}-\mathrm{cm}^{\wedge} 2$. The same ideal ID is moved to site $\mathrm{B}$. Now the $1^{\text {st }}$ integral changes by $216 \mathrm{G}-\mathrm{cm}$ as the gap is varied and the $2^{\text {nd }}$ integral will appear to change by $48,600 \mathrm{G}-\mathrm{cm}^{\wedge} 2$. We analyzed these effects in advance and pre-tuned the device for the ambient field at SRRC. When we arrived at SRRC we found that the normal and skew integrals agreed to within $10 \mathrm{G}-\mathrm{cm}$.

It is intuitively clear that at a small gap the ferromagnetic poles will shunt any skew ambient fields away from the e-beam and leave no on-axis skew field. Skew shunting will decrease as the gap is opened until it becomes absent for an infinite magnetic gap. Skew shunting is shown in Figure 2 where almost complete skew shunting can be seen at small gaps.

In the normal field direction, the field lines that would have gone through the magnets are shunted into the high permeability poles which leads to an overall normal field enhancement at small gaps. The field profiles for several gaps are shown in Figure 3. Skew and normal field effects after wiggle period averaging are summarized in Figure 4.

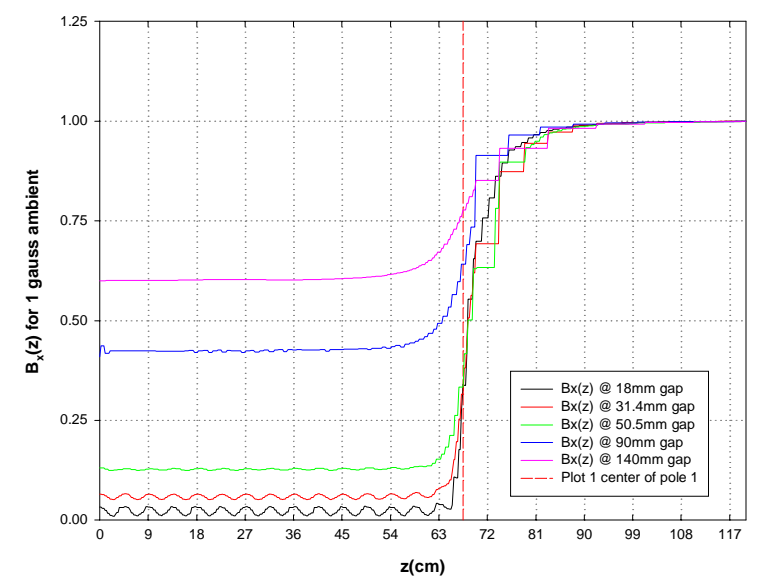

Figure 2. Skew field shunting at ends vs gap; gaps $=18 \mathrm{~mm}$ to $140 \mathrm{~mm}$

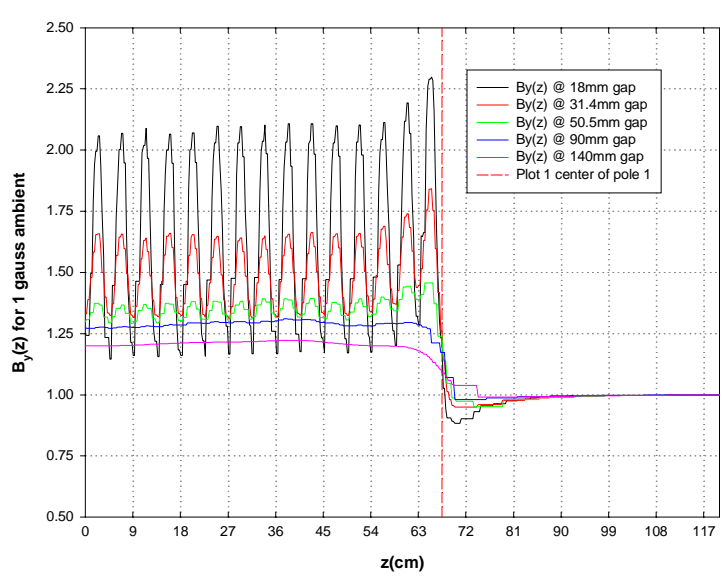

Figure 3. Normal field enhancement at ends vs gap; gaps $=18 \mathrm{~mm}$ to $140 \mathrm{~mm}$

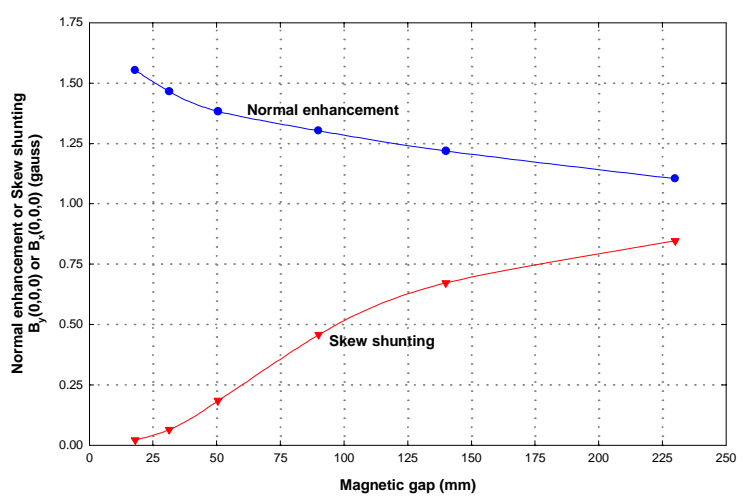

Figure 4. Gap dependence of centerline shunting effects, normal and skew fields.

\section{AIR GAP END CORRECTORS}

The U9 device is equipped with normal field, fixed gap, air core end correctors shown in Figure 5. If SRRC needs to actively point the photon beam, use of the control software can energize the correctors in a gap dependent manner. The coils have 49 turns and a rectangular shape with the steering legs are placed at \pm 30 degrees from vertical to eliminate sextupole moments. There is some loss in efficiency due to fringe fields being shunted into the first pole. The correctors did not introduce any significant multipole moments at their maximum currents of $10 \mathrm{amps}$. There are no skew field end correctors. 


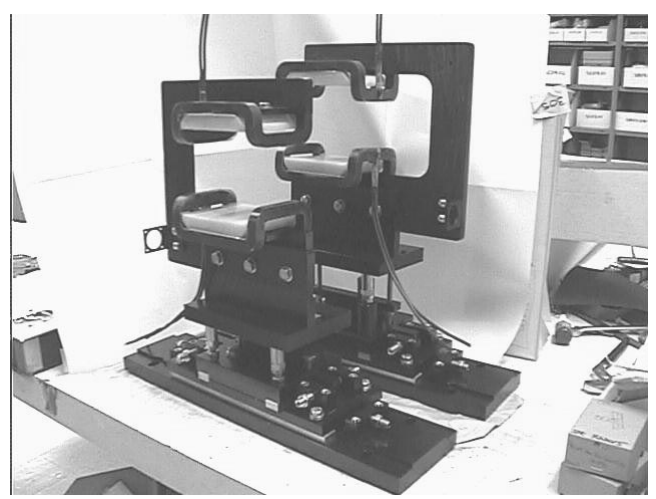

Figure 5. Air gap end corrector photo.

\section{STRONGBACK SHAPING TO REDUCE GAP DEPENDENT PHASE ERRORS}

For the U9 device there is a 12.5 metric ton variation in the attractive force between minimum and maximum gaps. Strongback deflections under magnetic loads will cause local gap variations which leads to a reduced spectral brightness. Spectral analyses for a collection of 25 previously built ID's having periods from $1.8 \mathrm{~cm}$ to $8.5 \mathrm{~cm}$ and $\mathrm{K}=11.9$ to 0.5 were used to test the Walker phase error model. ${ }^{6}$ The model did an excellent job of predicting the average harmonic intensity loss caused by phase errors. This allowed us to predict phase errors based on local gap variations due to strongback deflections vs gap.

The ideal situation is to eliminate any deflection by using large, heavy strongbacks. For U9 there were overall height and weight restrictions. We used stainless steel strongbacks rather than aluminum and devised a new 4 point beam hanger system to reduce the deflection to 25 microns. This produced the gap dependent phase error variation of 2 degrees which would produce a $1.0 \%$ loss in $3^{\text {rd }}$ harmonic intensity.

Experimental phase error plots showed that strongback deflection under load was responsible for almost all of the non-ideal spectral performance. The remaining spectral error sources were several micron gap dependent tapers ( $<0.5$ degrees) and field errors.

\section{CONCLUSION}

The SRRC/U9 met all magnetic requirements. It used side magnets to increase the on-axis field without any performance degradation. Ambient field correction succeeded in predicting changes to 1 st and 2 nd integrals and internal trajectories due to gap dependent difference in the ambient fields at STI and SRRC. The Walker phase error model ${ }^{6}$ was verified on an ensemble of 25 APS ID's having $\lambda_{\mathrm{w}}=18 \mathrm{~mm}$ through $85 \mathrm{~mm}$ and was used to specify strongback deflection.

\section{ACKNOWLEDGEMENTS}

The authors would like to acknowledge the contributions of the employees of STI to the construction and measurements of the U9/SRRC device. The advise of T.C. Fan, C.-H. Chang, C.-S. Hwang and J. Chen of SRRC are sincerely appreciated.

\section{REFERENCES}

[1] D.C. Quimby and A.L. Pindroh, "High Field Strength WedgedPole Hybrid Undulator," Rev. Sci. Instr. 58, pp. 339-345 (1987).

[2] S.C. Gottschalk, D.C. Quimby, K.E. Robinson, "ZeroDisplacement End Termination of Undulators and Wigglers," proceedings of this conference.

[3] D.C. Quimby, S.C. Gottschalk, D.R. Jander, T.E. DeHart, K.E. Robinson, A.S. Valla, and J.F. Zumdieck, "The 90-mm Period Undulator for SRRC," proceedings of this conference.

[4] S.C. Gottschalk, D.C. Quimby, K.E. Robinson, E.R. Moog, and R.J. Aparao, "Central Field Design Methods for Hybrid Insertion Devices," Rev. Sci. Instr. 67 (1996), Paper CO7 (CD-ROM issue).

[5] S.C. Gottschalk, K.E. Robinson, D.C. Quimby, K.W. Kangas, I. Vasserman, R. Dejus, and E. Moog, "Multipole and Phase Tuning Methods for Insertion Devices."

[6] B. Diviacco and R.P. Walker, "Recent Advances in Undulator Performance Optimization," Sincrotrone Trieste ST/M-95/3, Submitted to Nucl. Instrum. Meth., July 1995. See also B. Diviacco, et al., in the Proceedings of the 1993 IEEE Particle Accelerator Conf., Washington, D.C., 17-20 May 1993, IEEE Catalog No. 93CH3279-7, 1590 (1993). 